\title{
Design and modelling of all-optical NAND gate using metal-insulator-metal (MIM) waveguides based Mach- Zehnder Interferometers for high- speed information processing
}

Sandip Swarnakar ( $\nabla$ sandipswarnakar.2008@gmail.com )

G.Pulliah College of Engineering and Technology https://orcid.org/0000-0003-3767-0916

\section{Siva Koti Reddy}

Godavari Institute of Engineering and Technology

Ramanand Harijan

BM Group of Institutions

\section{Santosh Kumar}

Liaocheng University

\section{Research Article}

Keywords: Plasmonic waveguide, MIM waveguide, Mach-Zehnder interferometer, all-optical logic gate, finite-difference time-domain (FDTD)

Posted Date: April 19th, 2021

DOI: https://doi.org/10.21203/rs.3.rs-422938/v1

License: (9) This work is licensed under a Creative Commons Attribution 4.0 International License.

Read Full License

Version of Record: A version of this preprint was published at Optical and Quantum Electronics on August 14th, 2021. See the published version at https://doi.org/10.1007/s11082-021-03153-x. 


\title{
Design and modelling of all-optical NAND gate using metal-insulator-metal (MIM) waveguides based Mach- Zehnder Interferometers for high-speed information processing
}

\author{
Sandip Swarnakar ${ }^{a,}$, Siva Koti Reddy ${ }^{\mathrm{b}}$, Ramanand Harijan ${ }^{\mathrm{c}}$, Santosh Kumar ${ }^{\mathrm{d}, \mathrm{e}}$ \\ aPhotonics Lab, Department of Electronics and Communication Engineering, G. Pullaiah College of \\ Engineering and Technology, Nandikotkur Road, Kurnool, Andhra Pradesh -518002, India

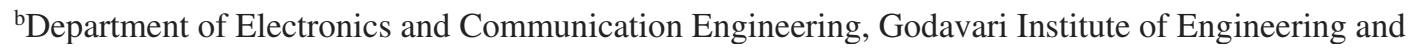 \\ Technology, Chaitanya Knowledge City, Rajahmundry-533296, Andhra Pradesh, India \\ ${ }^{c}$ Department of Electronics and Communication Engineering, BM Group of Institutions, Hari Nagar (Dumha), \\ Farrukh Nagar, Gurugram-122506, Haryana, India \\ ${ }^{\mathrm{d} D e p a r t m e n t ~ o f ~ E l e c t r i c a l ~ a n d ~ E l e c t r o n i c s ~ \& ~ C o m m u n i c a t i o n ~ E n g i n e e r i n g, ~ D I T ~ U n i v e r s i t y, ~ D e h r a d u n ~ 248009, ~}$ \\ Uttarakhand, India

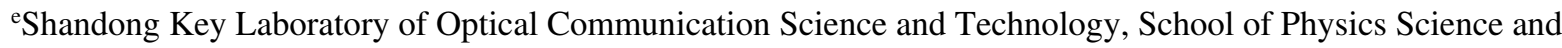 \\ Information Technology, Liaocheng University, Liaocheng - 252059, Shandong, China \\ *corresponding author: Email address: sandipswarnakar.2008@gmail.com
}

\begin{abstract}
All the basic logic gates play a major role in carrying out the mathematical computation. The drawbacks of conventional electronics are alleviated by all-optical integrated circuits with a great application of high-speed computing and information processing. In this paper, plasmonic metal-insulator-metal (MIM) waveguides have an excellent property of propagating the surface plasmons beyond the diffraction limit up to deep sub-wavelength scale. All-optical NAND gate design is optimized by using MIM plasmonic waveguide-based Mach-Zehnder Interferometers (MZIs) in the footprint of $36 \mu \mathrm{m} \times 8 \mu \mathrm{m}$ that works at 1.55 $\mu \mathrm{m}$ operating wavelength. The better performance of the proposed device is achieved, such as the extinction ratio is $10.55 \mathrm{~dB}$, insertion loss is obtained as $0.506 \mathrm{~dB}$, and response time is 262 ps. The proposed design is verified by using the finite-difference time-domain (FDTD) technique and further analysis are carried out by mathematical computation and MATLAB simulation results.
\end{abstract}

Keywords: Plasmonic waveguide; MIM waveguide; Mach-Zehnder interferometer; alloptical logic gate, finite-difference time-domain (FDTD) 


\section{Introduction}

In the earlier days, the enrichment of changing information is attained by semiconductor devices, though predictably used this semiconductor technology gets menial due to some restraints like heat dissipation and interconnect delays [1]. The telecommunication and network started research on photonics devices due to the increase of high deprivation of capacity and small size, in which the alteration of information is done through photons rather than electrons [2]. Due to miniaturization and with huge density devices, the diffraction of light becomes more susceptible. To overcome this problem, the restraining and regulating of light yonder diffraction of light can be contrived viable by using plasmonics, it attributes the combination of electrons and photonics. [3-4]. Due to some unique features, the propagation has been achieved by surface plasmon polaritons (SPPs) in plasmonics. The synergy of the electromagnetic waves and free electrons on the metals produce the surface plasmon polaritons which is a restrained wave that interface between the metal and dielectric [5]. In the last few years, the researchers lead to scrutiny of various plasmonic waveguides based on SPPs such as metal-insulator-metal (MIM), insulator-metal-insulator (IMI), dielectric-loaded surface plasmon polaritons (DLSPP) [6-10]. Among all these geometric waveguides, MIM waveguide has been preferred due to its their capability of exceptional properties like subwavelength confinement, very high grouping velocity and routing of light at nanoscale is also possible. By using semiconductor optical amplifier Mach- Zehnder interferometer (SOAMZI) [11-13] and lithium-niobate $\left(\mathrm{LiNbO}_{3}\right)$ [14-16] are already have been implemented for all the basic and universal logic gates. This work proposes the optimization of footprint in the NAND gate structure. As compared to the earlier design of the NAND gate using electrooptic effect, used four MZIs [17-19] to implement NAND gate but the proposed design is achieved by using only two MZIs to implement all-optical plasmonic NAND gate. Using these MIM waveguides, some designs are directional coupler (DC) and non-linear MachZehnder interferometers (MZIs) have been proposed and serving as a house for optical circuit designs. The significant phenomenon of directional coupler is the coupling of optical signals is beating for even-odd modes. By combining two directional couplers, an MZI is proposed with two linear waveguides in which one linear (linear 2) waveguide is filled with non-linear Kerr material to accomplish the exchange of light and named as nonlinear plasmonic MZI or,

metal-insulator-metal (MIM) waveguides based MZI. All-optical components have a 
remarkable and vital role in modern integrated and optical circuits in like a manner; all logic gates play a major role in the electronic devices.

The outline of this paper presents a design of all-optical universal NAND gate using MEH- PPV [poly (2-methoxy-5-(28-ethylhexyloxy)-PPV)] as a nonlinear Kerr [20] material in its one arm of plasmonics MZI. The analysis of the proposed structure is carried out by simulation in MATLAB and results are verified using finite-difference time-domain (FDTD) [21]. Section 2 presents a description of nonlinear single MZI and its mathematical description and portraying the propagating of light through it. Section 3 comprises of design and simulation results of the NAND gate. Finally, Section 4 comprises the conclusion of work.

\section{Design of metal-insulator-metal waveguides based Mach- Zehnder Interferometer}

In this paper, metal-insulator-metal waveguides based MZI is designed by cascading of two directional couplers and two linear metal-insulator-metal (MIM) waveguides in which one linear (linear 2) waveguide is filled with nonlinear Kerr material to achieve switching of light as shown in Fig 1.

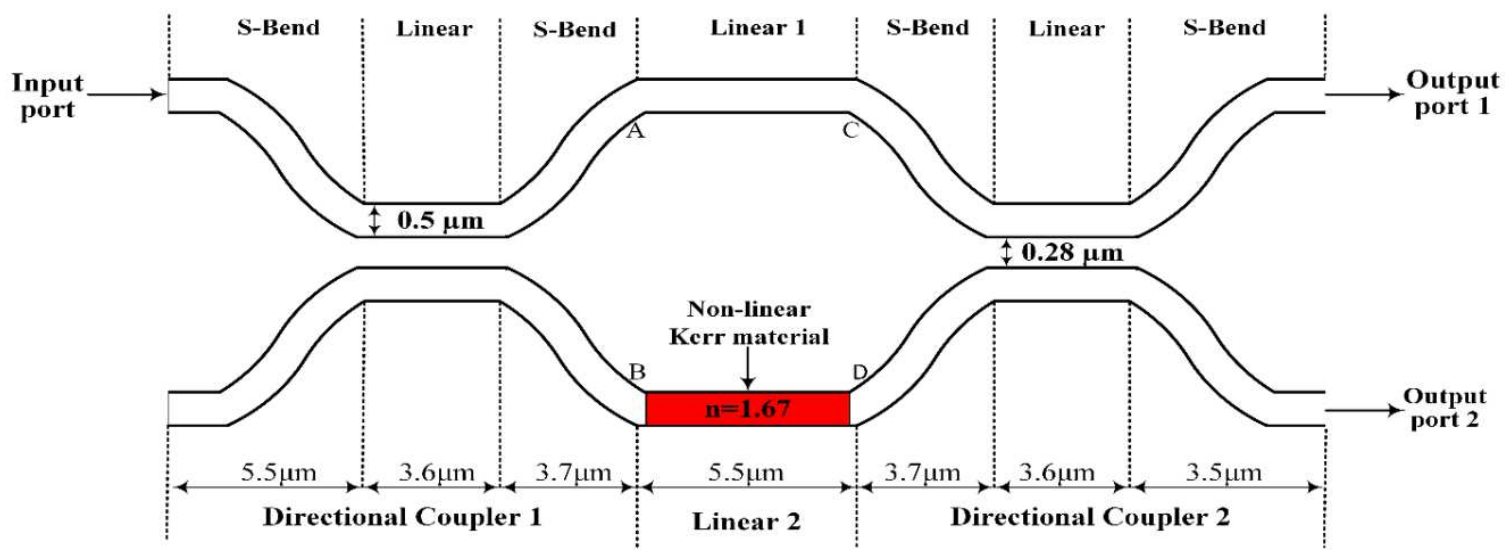

Fig. 1 Schematic of nonlinear Mach-Zehnder interferometer

The structure of MZI has a size within the footprints of $(32 \times 3.2) \mu \mathrm{m}$. This is the basic optical device used to a great extent for the development of logic gates. MEH-PPV [poly (2methoxy-5-(28-ethylhexyloxy)-PPV)] is used as the nonlinear Kerr material which gives enormous Kerr nonlinearity with permittivity of $2 \mathrm{e}^{-18} \mathrm{~m}^{2} / \mathrm{v}^{2}$ [22]. The relative linear permittivity $(\varepsilon \mathrm{L})$ of Kerr material is 2.7225 with refractive index $(\mathrm{RI})$ and response time are $\mathrm{n}=1.65$ and $\tau=2.0 \mathrm{e}-15 \mathrm{~s}$, respectively. The dispersion equation for three-layered MIM waveguide is given as [23]:

$\frac{k_{m}}{\epsilon_{m}}=-\frac{k_{d}}{\epsilon_{d}} \tanh \left(\frac{t}{2} t_{d}\right)$ 
whereas $\epsilon_{\mathrm{d}}$ and $\epsilon_{\mathrm{m}}$ are the permittivity of dielectric and metal, respectively. $\mathrm{k}_{\mathrm{d}}$ and $\mathrm{k}_{\mathrm{m}}$ are the transverse wave number for dielectric and metal, respectively; where ' $d$ ' is the width of the linear insulator, which is taken as air. By using a Drude Lorentz model, the metal supporting SPPs are considered as silver [24]:

$\epsilon_{2}(\omega)=1-\frac{\omega_{p}^{2}}{\omega(\omega+i \gamma)}+\sum_{n=1}^{5} \frac{f_{n \omega_{n}^{2}}}{\omega_{n}^{2}-\omega^{2}-i \omega \gamma n}$

$\omega_{\mathrm{p}}=140 \times 1014 \mathrm{rad} / \mathrm{s}$ and $\gamma=0.13 \times 1014 \mathrm{rad} / \mathrm{s}$ are bulk plasma frequency of silver and damping constant, respectively. By changing the RI of Kerr material, the intensities of light vary, which is called the Kerr effect, and the phase of the signal varies, which leads to switching of the light signal from one port to another port by a change in power of input signal. When continuous wave $(\mathrm{CW})$ of wavelength $1550 \mathrm{~nm}$ excites transverse electric (TE) mode with an input power of $\mathrm{E}_{\mathrm{in}}=3 \mathrm{e}^{9} \mathrm{~W} / \mathrm{m}$ (considered as logic ' 1 ') is given at input port 1 , by obeying the principle of self-phase modulation (SPM), output signal obtained at the first output port as shown in Fig. 2(b). When input power of $\mathrm{E}_{\mathrm{in}}=0.7 \mathrm{e}^{9} \mathrm{~W} / \mathrm{m}$ (considered as logic ' 0 ') is given at input port 1 , by obeying the principle of cross-phase modulation (XPM), output signal obtained at second output port as shown in Fig. 2(c).

If an input, $E_{\text {in }}$ applied at input port, the output at A and B can be written [25-26] as:

$\left.A=\sqrt{1-\alpha_{1}}\left(E_{i n}\right)+j \sqrt{\alpha_{1}}(0)\right)$

$\left.B=j \sqrt{\alpha_{1}}\left(E_{\text {in }}\right)+\sqrt{1-\alpha_{1}}(0)\right\}$

where $\alpha_{1}$ is the attenuation constant of first directional coupler shown in Fig. 2(a) near input ports Eq. 3 can be written as:

$\left[\begin{array}{l}A \\ B\end{array}\right]=\left[\begin{array}{cc}\sqrt{1-\alpha_{1}} & j \sqrt{\alpha_{1}} \\ j \sqrt{\alpha_{1}} & \sqrt{1-\alpha_{1}}\end{array}\right]\left[\begin{array}{c}E_{\text {in }} \\ 0\end{array}\right]$

Further propagation of signal is from first directional coupler off from points A and B through linear arms reaches at point $\mathrm{C}$ and $\mathrm{D}$ at output ports respectively, the input signal can be written as

$c=A e^{-j \varphi_{1}}, D=B e^{-j \varphi_{2}}$

whereas $\varphi_{2}$ has a phase difference in second linear arm due to Kerr material and $\varphi_{1}$ has a value equal to zero since in the second linear arm there is no phase difference occurs as detailed in [27] as:

$\varphi_{1}=\Delta \varphi=(2 \pi / \lambda)\left(\widetilde{n_{X}}-\widetilde{n_{Y}}\right) L$

where $\lambda$ is the wavelength of signal and L is the length of the second linear arm of MZI and

$\left.\widetilde{n_{X}}=n_{X}+\Delta n_{X}\right\}$ 
where $\Delta \mathrm{x}$ and $\Delta \mathrm{y}$ are the linear different RIs in the second linear arms of the MZI, because of modal birefringence with low and high intensities of a signal, respectively. $\Delta n_{X}$ and $\Delta n_{Y}$ are the RIs of nonlinear parts because of the induced birefringence. If the signal is launched with low intensity, then

$\Delta n_{X}=2 n_{2}\left|E_{\text {in }}\right|^{2}$

where $E_{\text {in }}$ is the intensity of input signal. When high intensity signal is launched, then due to XPM, the RI becomes

$\Delta n_{Y}=2 n_{2} b\left|E_{\text {in }}\right|^{2}, b=\chi_{X X Y Y}^{(3)} / \chi_{X X X X}^{(3)}$

where $\mathrm{n}_{2}$ is the RI of the linear arm of the MZI containing Kerr material, $\chi_{X X Y Y}^{(3)}$ and $\chi_{X X X X}^{(3)}$ are the third-order susceptibility of the nonlinear Kerr material, $b=1 / 3$ where the signal is purely electronic. Using Eqs. (4) and (7), phase shift becomes;

$\varphi_{2}=\Delta \varphi_{L}+\Delta \varphi_{N L}=(2 \pi L / \lambda)\left(\Delta n_{L}+n_{2 B}\left|E_{\text {in }}\right|^{2}\right)$

where $\Delta n_{L}=n_{X}-n_{Y}$ and the Kerr coefficient is $\mathrm{n}_{2 \mathrm{~B}}=2 \mathrm{n}_{2}(1-\mathrm{b})$. For high signal intensity, $(\Delta \varphi \neq 0)$ and the maximum, the transmission of signal through a first linear arm of MZI, with transmitivity written as

$\mathrm{T}_{\text {out } 1}=\frac{1}{4}\left|1-e^{i \varphi 2}\right|^{2}=\sin ^{2}\left(\frac{\Delta \varphi}{2}\right)$

When low intensity of light signal is fed into the input, it arrives at the output port can be written as

$T_{\text {out } 2}=\cos ^{2}\left(\frac{\Delta \varphi}{2}\right)$

Eqs. (9) and (10) gives the output of single MZI, one can calculate the power when high and low-intensity signal is fed at its first input port, respectively. This exclusive switching property of MZI has been used to design the proposed device.

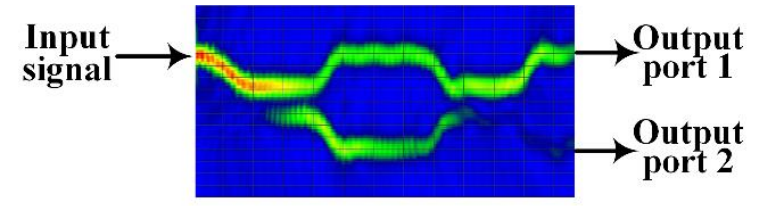

(a)

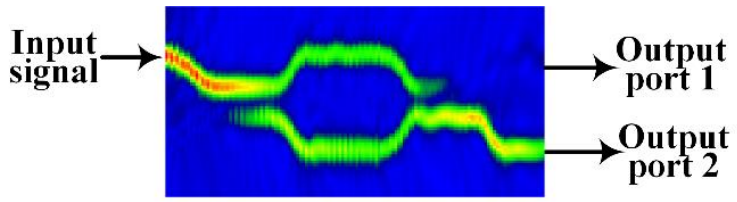

(b)

Fig. 2 Polarization of light through single MZI, at (a) high-intensity signal, (b) low-intensity signal

\section{Design, mathematical formulation and simulation results of all-optical NAND gate}

The miniature of all-optical NAND gate is designed using two nonlinear MZI within the footprint of $36 \mu \mathrm{m} \times 8 \mu \mathrm{m}$ as shown in Fig. 3. By using MIM, the proposed structure is 
designed which supports the plasmonic modes. To get desired operation of universal gate, the optical signals are given at first and second input port of MZI1 and MZI2, respectively. The second and first output ports of MZI1 and MZI2 provides phase shift leads to switching of light. To obtain the optical power at the output port, the two signals combined to get the output of the NAND gate.

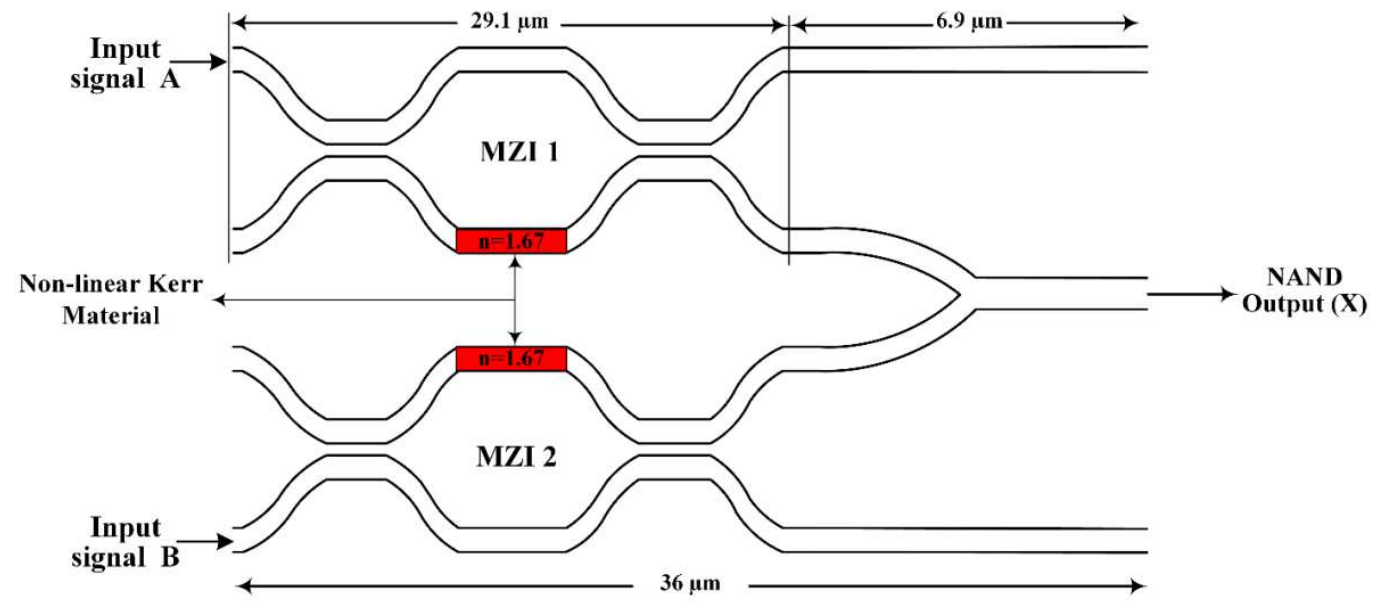

Fig. 3 Schematic representation of all-optical NAND gate

The normalized power for output of NAND gate can be calculated for possible combinations as follows [28]:

$$
\left.\begin{array}{ll}
m_{1}=\left[\cos ^{2}\left(\frac{\Delta \varphi M Z I 1}{2}\right)+\cos ^{2}\left(\frac{\Delta \varphi M Z I 2}{2}\right)\right] & \text { for } \mathrm{AB}=00 \\
m_{2}=\left[\cos ^{2}\left(\frac{\Delta \varphi M Z I 1}{2}\right)+\sin ^{2}\left(\frac{\Delta \varphi M Z I 2}{2}\right)\right] & \text { for } \mathrm{AB}=01 \\
m_{3}=\left[\sin ^{2}\left(\frac{\Delta \varphi M Z I 1}{2}\right)+\cos ^{2}\left(\frac{\Delta \varphi M Z I 2}{2}\right)\right] & \text { for } \mathrm{AB}=10 \\
m_{4}=\left[\sin ^{2}\left(\frac{\Delta \varphi M Z I 1}{2}\right)+\sin ^{2}\left(\frac{\Delta \varphi M Z I 2}{2}\right)\right] & \text { for } \mathrm{AB}=11
\end{array}\right\}
$$

Hence the overall output of NAND gate is OUTPUTNAND $=m_{1}+m_{2}+m_{3}$, because there is no output signal for the remaining combinations of input signals.

$$
\begin{aligned}
& \text { OUTPUT }_{\mathrm{NAND}}=\left[\cos ^{2}\left(\frac{\Delta \varphi M Z I 1}{2}\right)+\cos ^{2}\left(\frac{\Delta \varphi M Z I 2}{2}\right)+\cos ^{2}\left(\frac{\Delta \varphi M Z I 1}{2}\right)+\sin ^{2}\left(\frac{\Delta \varphi M Z I 2}{2}\right)+\right. \\
& \left.\sin ^{2}\left(\frac{\Delta \varphi M Z I 1}{2}\right)+\cos ^{2}\left(\frac{\Delta \varphi M Z I 2}{2}\right)\right]
\end{aligned}
$$

The design of the all-optical NAND gate is verified using MATLAB and FDTD method. By cascading two MZIs, the layout of all-optical NAND gate as shown in Fig. 3. A continuouswave $(\mathrm{CW})$ in transverse electric (TE) a source of wavelength $1550 \mathrm{~nm}$ is fed with both input power of $0.7 \mathrm{e}^{9} \mathrm{~W} / \mathrm{m}$ and $3 \mathrm{e}^{9} \mathrm{~W} / \mathrm{m}$ are considered as low and high- intensity optical signals, respectively. The proposed design is within the mesh size of $\Delta \mathrm{x}=0.07711 \mu \mathrm{m}$ and $\Delta \mathrm{z}=$ $0.0772 \mu \mathrm{m}$. The perfect matched layer (PML) is used as the boundary conditions, with the reflection coefficient of $\mathrm{e}^{-12}$. The propagation of the optical signal through the proposed 
design for all possible combinations of input signals is shown in Fig. 4. The timing diagram of the NAND gate is obtained through MATLAB simulation as shown in Fig. 5 is verified with the truth table as shown in Table.1. The different possible input combinations to verify the logic of NAND gate have been applied and discussed as per the following case study. Some operating parameters of plasmonic NAND gate are shown in Table. 2.

Table. 1: Truth table of NAND gate

\begin{tabular}{|c|c|c|}
\hline Input A & Input B & Output (X) \\
\hline 0 & 0 & 1 \\
\hline 0 & 1 & 1 \\
\hline 1 & 0 & 1 \\
\hline 1 & 1 & 0 \\
\hline
\end{tabular}

Table. 2: Operating parameters of proposed all-optical NAND gate

\begin{tabular}{|l|l|}
\hline Input field transverse & Gaussian \\
\hline Wavelength $(\lambda)$ & $1.55 \mu \mathrm{m}$ \\
\hline Polarization & Transverse electric \\
\hline No. of Mesh cells X & 64 \\
\hline No. of Mesh cells Z & 414 \\
\hline Boundary conditions & Perfectly matched layers \\
\hline Coupling length $\left(\mathrm{L}_{c}\right)$ & $3.6 \mu \mathrm{m}$ \\
\hline
\end{tabular}

\section{Case (a): $A=0, B=0$}

In this case, $A=0, B=0$, means both signals are low-intensity signals fed from the first and second ports of MZI1 and MZI2. The outputs of these MZIs have arrived at the opposite port of the same MZI. The outputs are combined due to SPM and thereafter the output signal arrives at the output port $(\mathrm{X})$ is considered as the output of this combination. Thus, the output of the NAND gate is " 1 " (as shown in Fig.4 (a)).

\section{Case (b): $A=0, B=1$}

At this point, $A=0$ means low- intensity signal is fed from first input port of MZI1, due to SPM, the output signal arrives at the cross port of the same MZI1. For B=1 means highintensity signal is fed from second input port of MZI2, due to XPM, the output signal arrives at the first output port of the same MZI2. At the output port (X), a signal from the MZI1 is 
considered as the output of this combination. Thus, the output of NAND gate is "1" (as shown in Fig. 4 (b)).

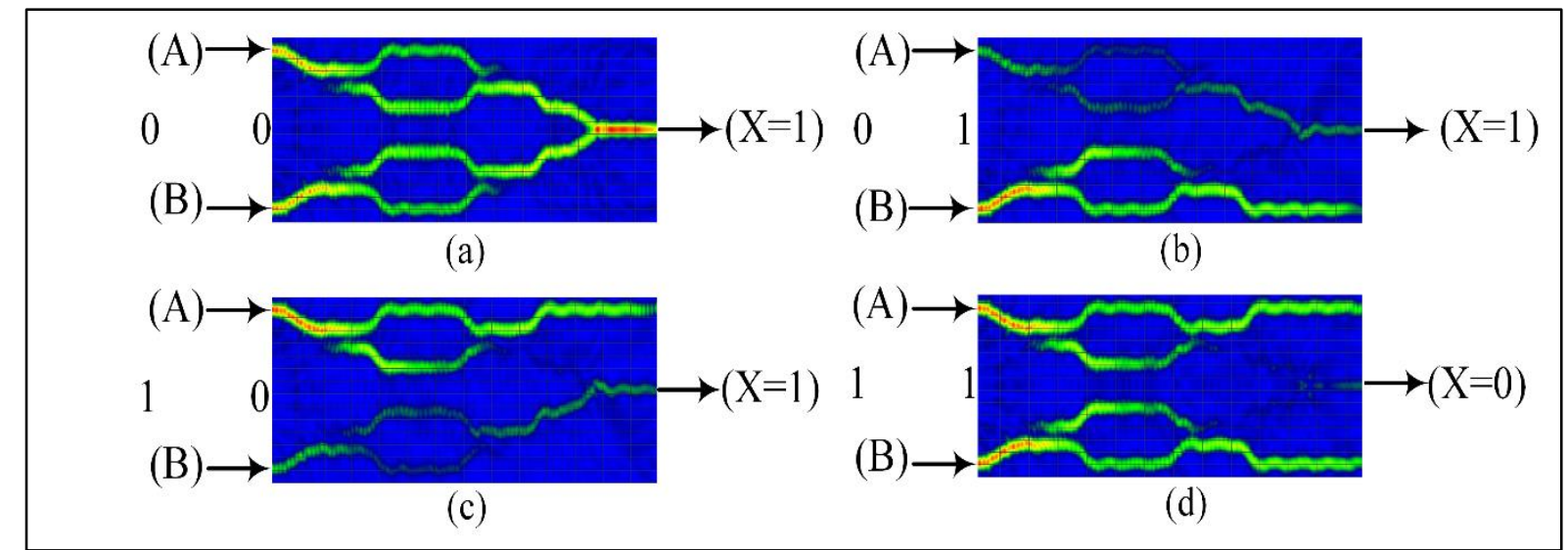

Fig. 4 Output results for all possible combinations of an optical signal obtained from FDTD Case (c): $A=1, B=0$

Under this case, $A=1$ means high- intensity signal is fed from the first input port of MZI1, due to XPM, the output signal arrives at the first output port of the same MZI1. For B=0 means low- intensity signal is fed from the second input port of the MZI2, due to SPM, the output signal arrives at the first output port the same MZI2. At the output port (X) signal from the MZI2 is considered as the output of this combination. Thus, the output of NAND gate is "1" (as shown in Fig.4 (c))

\section{Case (d): $A=1, B=1$}

For this case, $A=1$ means high- intensity signal is fed from first input port of MZI1, due to XPM, the output signal arrives at the first output port of the same MZI1. For $B=1$ means high- intensity signal is fed from the second input port of the MZI2, due to XPM, the output signal arrives at the second output port of the same MZI2. At the output port (X) no signal has arrived from both MZIs. Thus, the output of NAND gate is "0" (as shown in Fig.4 (d)). From the above-discussed cases, we observed that the output of NAND gate is obtained only when both or either one of the inputs is fed with low intensity, which is exactly matched with the MATLAB timing diagrams shown in Fig. 5. 


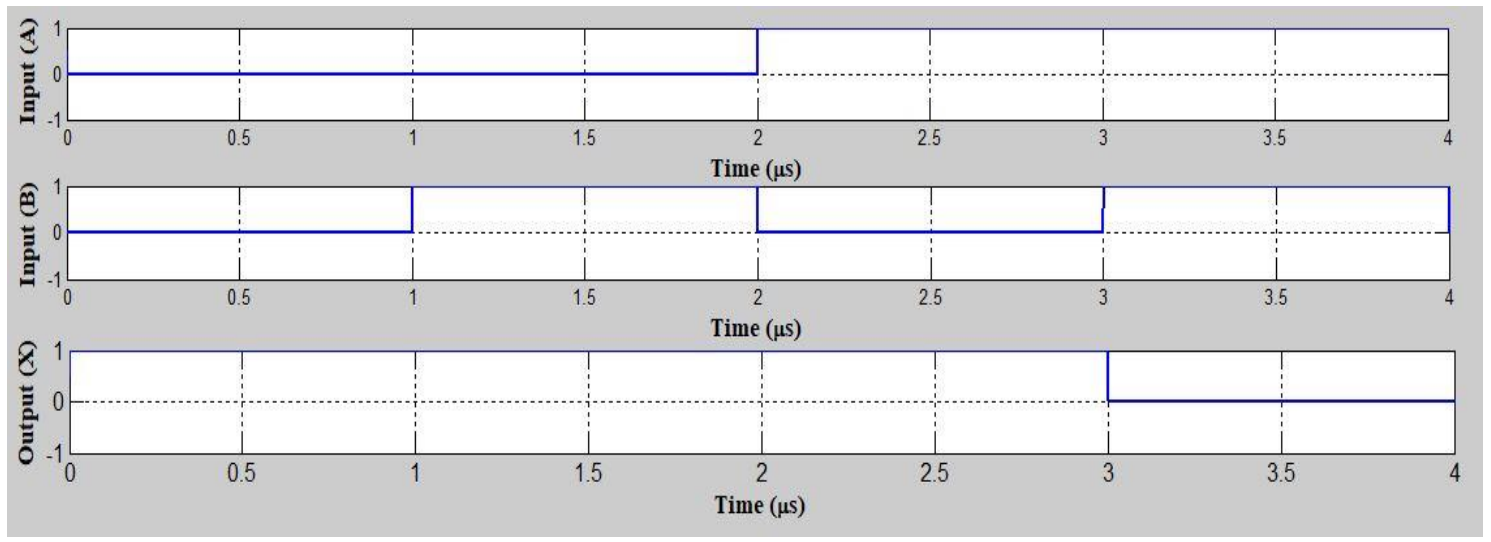

Fig. 5 MATLAB simulation results for all-optical NAND gate

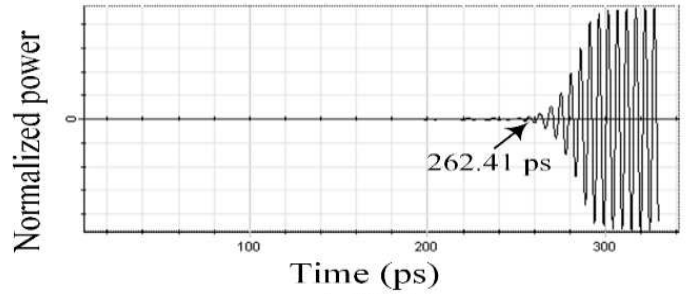

a) " 00 "

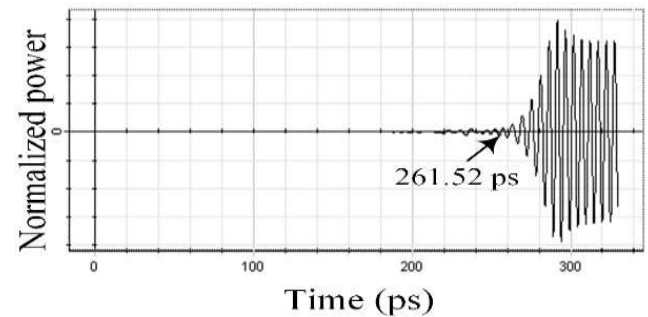

b) "01"

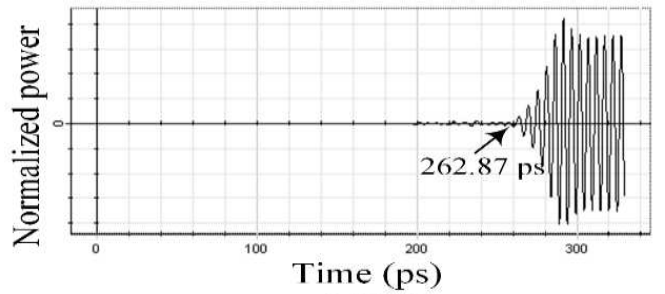

c) " 10 "

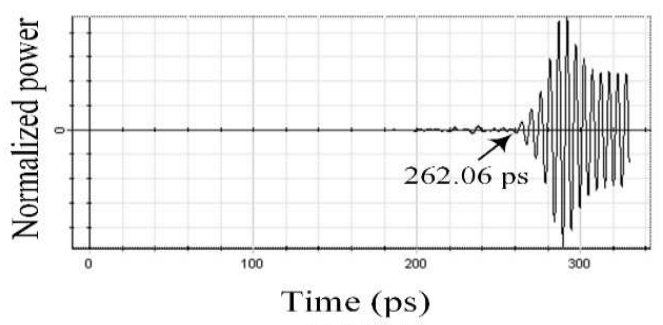

d) " 11 "

Fig. 6 Normalized power versus time for all four combinations: a) " 00 ”; b) “01"; c) "10";

$$
\text { d) " } 11 \text { " }
$$

The response time is calculated for all the four input combinations of the proposed all-optical plasmonic NAND gate. The output power level requires some time to become stable. In the NAND gate for all the combinations, the response time almost equal. The response time of the proposed design all-optical NAND gate is in the range of 261.52 ps to 262.87 ps. The response time for all four combinations is shown in Fig. 6.

Table. 3: Performance study of proposed logic gate

\begin{tabular}{|c|c|c|c|}
\hline Sl. No. & Parameters & Formulae & Value (dB) \\
\hline 1. & $\begin{array}{l}\text { Extinction } \\
\text { ratio }\end{array}$ & 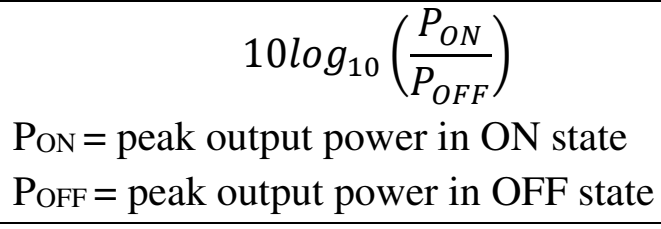 & 10.55 \\
\hline
\end{tabular}




\begin{tabular}{|l|c|l|l|}
\hline 2. & $\begin{array}{c}\text { Insertion } \\
\text { loss }\end{array}$ & \multicolumn{1}{c|}{$\begin{array}{c}10 \log _{10}\left(\frac{P_{\text {in }}}{P_{\text {out }}}\right) \\
P_{\text {in }}=\text { peak input power } \\
P_{\text {out }}=\text { peak output power }\end{array}$} & 0.506 \\
\hline
\end{tabular}

The performance analysis of the all-optical plasmonic NAND logic gate is studied by calculating some parameters like extinction ratio (ER) and insertion loss (IL) are provided in Table. 3. The ER is calculated as $10.55 \mathrm{~dB}$ and IR is observed as $0.506 \mathrm{~dB}$. The performance and some key design parameters of the proposed design is compared with the earlier designs are shown in Table. 4. The proposed all-optical plasmonic NAND gate is compared with existing plasmonic NAND gate, NAND gate designed by using semiconductor optical amplifier (SOA), and photonic crystal ring resonator. From Table. 4, we can easily determine that the proposed structure has less size as compared to previous design and the extinction ratio and insertion loss are also optimized.

Table 4: Performance and design parameter comparison of proposed all-optical plasmonic NAND gate with existing devices

\begin{tabular}{|c|c|c|c|c|c|}
\hline $\begin{array}{l}\text { Sl. } \\
\text { No. }\end{array}$ & Parameters & $\begin{array}{c}\text { Semiconductor } \\
\text { Optical } \\
\text { Amplifier } \\
\text { (SOA) NAND } \\
\text { gate Ref. [29] }\end{array}$ & $\begin{array}{c}\text { Existing } \\
\text { photonic crystals } \\
\text { NAND gate } \\
\text { Ref. }[30]\end{array}$ & $\begin{array}{c}\text { Existing } \\
\text { plasmonic all- } \\
\text { optical NAND } \\
\text { gate Ref. [31] }\end{array}$ & $\begin{array}{l}\text { Proposed } \\
\text { plasmonic } \\
\text { all-optical } \\
\text { NAND gate }\end{array}$ \\
\hline 1. & Dimensions & - & $43.75 \mu \mathrm{m} \times 37.625 \mu \mathrm{m}$ & $40 \mu \mathrm{m} \times 7.5 \mu \mathrm{m}$ & $36 \mu \mathrm{m} \times 8 \mu \mathrm{m}$ \\
\hline 2. & Wavelength & - & $1.55 \mu \mathrm{m}$ & $1.55 \mu \mathrm{m}$ & $1.55 \mu \mathrm{m}$ \\
\hline 3. & Polarization & - & TM & $\mathrm{TE}$ & $\mathrm{TE}$ \\
\hline 4. & $\begin{array}{c}\text { Extinction } \\
\text { ratio }\end{array}$ & $9.73 \mathrm{~dB}$ & $20 \mathrm{~dB}$ & $10.25 \mathrm{~dB}$ & $10.55 \mathrm{~dB}$ \\
\hline 5. & $\begin{array}{c}\text { Insertion } \\
\text { loss }\end{array}$ & - & - & $0.756 \mathrm{~dB}$ & $0.506 \mathrm{~dB}$ \\
\hline
\end{tabular}

\section{Conclusion:}

In this paper, metal-insulator-metal (MIM) plasmonic waveguides are used to design the Mach- Zehnder interferometer (MZI). An MZI switching property is mainly used to design an all-optical NAND gate. The footprint of the designed gate is $36 \mu \mathrm{m} \times 8 \mu \mathrm{m}$ at an operating wavelength of $1.55 \mu \mathrm{m}$. Some parameters are calculated like extinction ratio (ER) and 
insertion loss (IL) are $10.55 \mathrm{~dB}$ and $0.506 \mathrm{~dB}$, respectively. The response time of the proposed all-optical NAND gate is in the range of 261.52 ps to 262.87 ps. As per Table 4, performance of the proposed gate is better than the earlier NAND gate. The study of the proposed design verification is done using mathematical computation, MATLAB simulation and carried out by FDTD results.

\section{Acknowledgment}

This work was supported by the Science and Engineering Research Board, India [grant number: TAR/2018/000051].

\section{Disclosure}

The authors declare that there are no conflicts of interest related to this article.

\section{References}

[1] Cotter, D., Manning, R.J., Blow, K.J., Ellis, A.D., Kelly, A.E., Nesset, D., Phillips, I.D., Poustie, A.J., Rogers, D.C.: Nonlinear optics for high-speed digital information processing. Sci. 286(5444), 1523-1528 (1999)

[2] Wu, Y.D.: Nonlinear all-optical switching device by using the spatial soliton collision. Fib. and Integ. Opt. 23(5), 387-404 (2004)

[3] Gibbs, H.M.: Optical bi-stability: Controlling Light with Light. 1st edn. Academic Press, New York, $1^{\text {st }}$ Edi. (1985)

[4] Hu, X., Jiang, P., Ding, C., Yang, H., Gong, Q.: Picosecond and low-power all-optical switching based on an organic photonic-bandgap micro-cavity. Nat. Photon. 2(3), 185189 (2008)

[5] Bernes, W.L., Dereux, A., Ebbesen, T.W.: Surface plasmon subwavelength optics. Nat. 424, 824-830 (2003)

[6] Zia, R., Schuller, J.A., Chandran, A., Brongersma, M.L.: Plasmonics: the next chipscale technology. Mater. Today. 9(7), 20-27 (2006)

[7] Dionne, J.A., Sweatlock, L.A., Atwater, H.A., Polman, A.: Plasmon slot waveguides: Towards chip scale propagation with subwavelength-scale localization. Phys. Rev. B. 73(3), 035407-035416 (2006)

[8] Pile, D.F.P, Ogawa, T.: Two-dimensionally localized modes of a nanoscale gap plasmon waveguide. Appl. Phys. Lett. 87(26), 261114-261116 (2005) 
[9] Chen, Z., Holmgaard, T., Bozhevolnyi, S.I., Krasavin, A.V., Zayats, A.V., Markey, L, Dereux, A.: Wavelength-selective directional coupling with dielectric-loaded plasmonic waveguides. Opt. Lett. 34(3), 310-312 (2009)

[10] Charbonneau, R., Lahoud, N., Mattiussi, G., Berini, P.: Demonstration of integrated optics elements based on long-ranging surface plasmon polaritons. Opt. Exp. 13(3), 977-984 (2005)

[11] Datta, K., Chattopadhyay, T., Sengupta, I.: All optical design of binary adders using semiconductor optical amplifier assisted Mach-Zehnder interferometer. Microelectron. 46(9) 839-847 (2015)

[12] Kim, J.Y., Kang, J.M., Kim, T.Y., Han, S.K.: 10 Gbit/s all-optical composite logic gates with XOR, NOR, OR and NAND functions using SOA-MZI structures, Electron. Lett. 42(5) 303- 304 (2006)

[13] Samanta, D., Mukhopadhyay, S.: All-optical method of developing parity generator and checker with polarization encoded light signal. J. Opt. 41(3), 167-172 (2012)

[14] Wooten, E.L., Kissa; K.M., Yan, A.Y., Murphy, E.J., Lafaw, D.A., Hallemeier, P.F., Maack, D., Attanasio, D.V., Fritz, D.J., McBrien, G.J., Bossi, D.E.: A Review of Lithium Niobate Modulators for Fiber-Optic Communications Systems. IEEE J. Selected Topics Quant. elect. 6(1), 69- 82 (2000)

[15] Zhang, M., Chen, K., Jin, W., Chiang, S.: Electro-optic mode switch based on lithiumniobate Mach -Zehnder interferometer. Appl. Opt. 55(16) 4418-4422 (2016)

[16] Takeda, K., Hoshina, T., Takeda, H., Tsurumi, T.: Electro-optic effect of lithium niobate in piezoelectric resonance. J. Appl. Phys. 112(12), 1-6 (2012)

[17] Smith, D.S., Riccius, H.D., Edwin, R.P.: Refractive indices of lithium niobate. Opt. Commun. 17(3), 332-335 (1976)

[18] Kumar, S., Singh, L., Chen, N.K.: Design of all-optical universal gates using plasmonics Mach-Zehnder interferometer for WDM applications. Plasmon. 13, 1277$1286(2018)$

[19] Yu, J., Zhang, J.: Recent progress on high-speed optical transmission. Digit. Commun. Netw. 2(2), 65-76 (2016)

[20] Pal, A., Kumar, S., Sharma, S., Raghuwanshi, S.K.: Design of 4 to 2 line encoder using lithium niobate based Mach Zehnder Interferometers for high speed communication. Proc. of SPIE - Opt. Model. and Design IV. SPIE Photon. Europe 9889, 1-6 (2016) https://doi.org/10.1117/12.2225867 
[21] Scheuer, J., Orenstein, M.: All-optical gates facilitated by soliton interactions in a multilayered Kerr medium. J. Opt. Soc. Am. B 22(6), 1260-1267 (2005)

[22] Pereda, J.A., Vegas, A., Prieto, A.: An improved compact 2D full-wave FDTD method for general guided wave structures. microw. Opt Tech. Lett. 38(4), 331-335 (2003)

[23] Bader, M.A., Marowsky, G., Bahtiar, A., Koynov, K., Bubeck, C., Tillmann, H., Hörhold, H.H., Pereira, S.: Poly(p-phenylenevinylene) derivatives: New promising materials for nonlinear all-optical waveguide switching. J. of Opt. Soc. of Ame. B. 19(9), 2250-2262 (2002)

[24] Pile, D.F.P., Ogawa, T., Gramotnev, D.K., Matsuzaki, Y., Vernon, K.C., Yamaguchi, K., Okamoto, T., Haraguchi, M., Fukui, M.: Two dimensionally localized modes of a nanoscale gap plasmon waveguide. Appl. Phys. Lett. 87(26), 1-3 (2005)

[25] Pannipitiya, A., Rukhlenko, I.D., Premaratne, M., Hattori, H.T., Agrawal, G.P.: Improved transmission model for metal-dielectric-metal plasmonic waveguides with stub structure. Opt. Exp. 18(6), 6191- 6204 (2010)

[26] Kumar, S., Singh, L.: Proposed new approach to design all optical AND gate using plasmonic based Mach-Zehnder interferometer for high speed communication. Proc. of SPIE, Nanophoton. VI, Eup. 9884, 1-7 (2016) https://doi.org/10.1117/12.2227069

[27] Raghuwanshi, S.K., Kumar, A., Kumar, S.: $1 \times 4$ signal router using three MachZehnder interferometers. Opt. Engg. 52(3), 1-9 (2013)

[28] Agrawal, G.P.: Nonlinear fiber optics. 3rd edn. Academic Press, New York 2006

[29] Mehra, R., Jaiswal, S., Dixit, H.K.: SOA based all optical gate and their comparison.

IEEE 2012 3rd Int. Conf. Compu. Commun. Tech. (ICCCT). 175- 177 (2012) https://doi.org/10.1109/ICCCT.2012.42

[30] Fu, Y., Hu, X., Gong, Q.: Silicon photonic crystal all-optical logic gates. Phys. Lett. A. 377(3-4), 329-333 (2013)

[31] Singh, A., Pal, A., Singh, Y., Sharma, S.: Design of optimized all-optical NAND gate using metal-insulator-metal waveguide. Opt. Int. J. for Light and Elect. Opt. 182(7), $524-528(2019)$ 
Figures

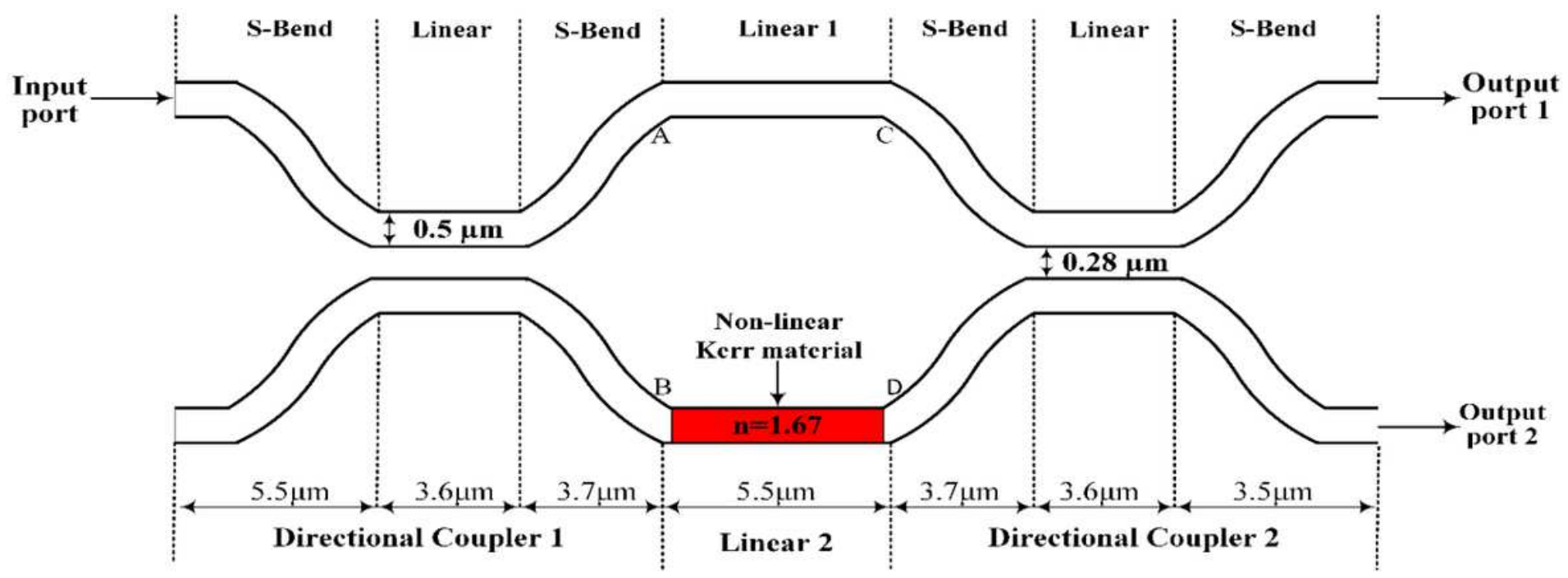

Figure 1

Schematic of nonlinear Mach-Zehnder interferometer

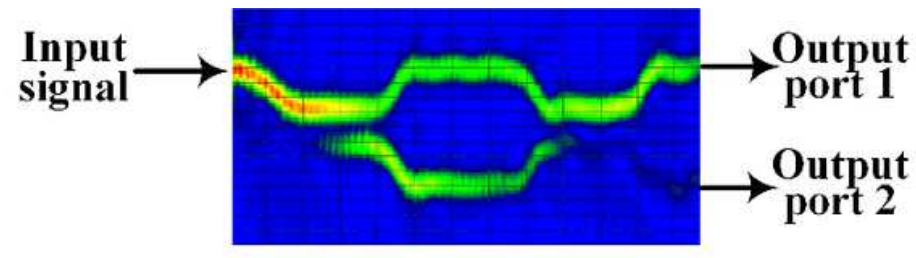

(a)

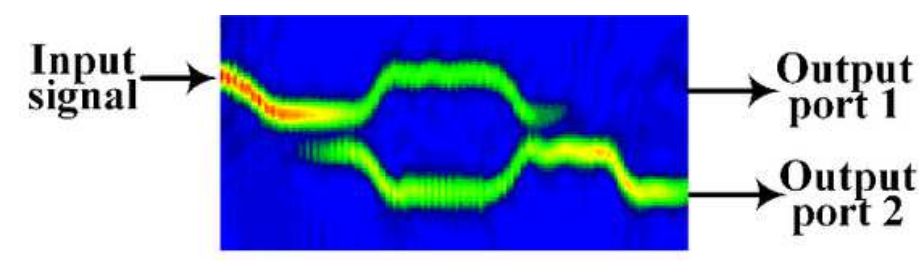

(b)

Figure 2

Polarization of light through single MZI, at (a) high-intensity signal, (b) low-intensity signal

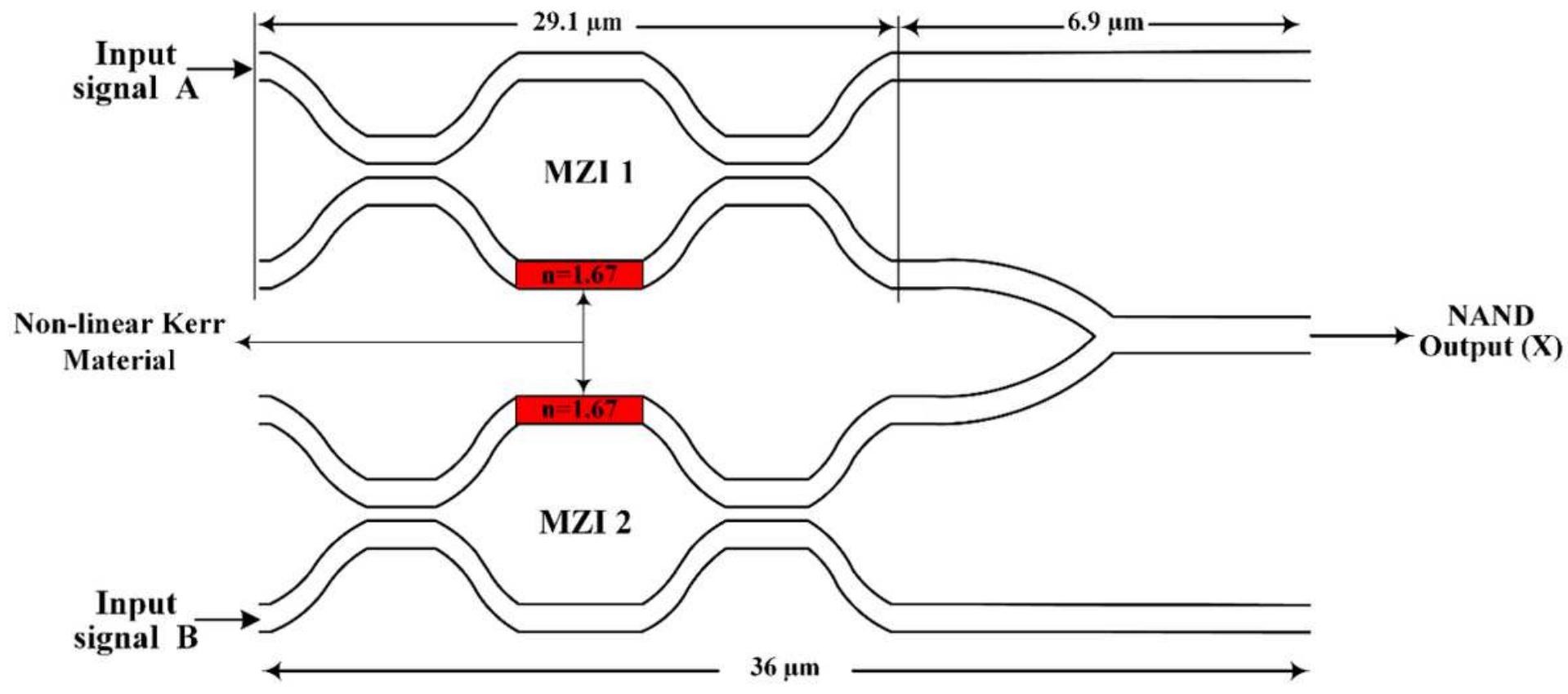


Figure 3

Schematic representation of all-optical NAND gate

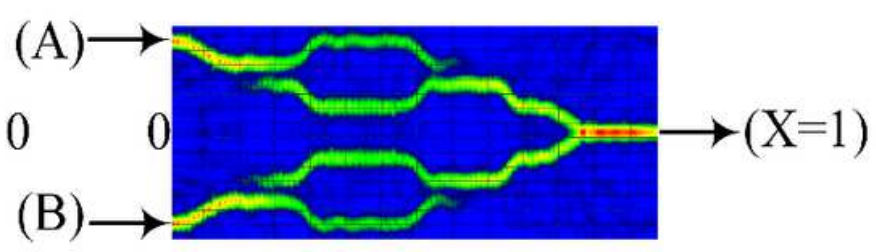

(a)

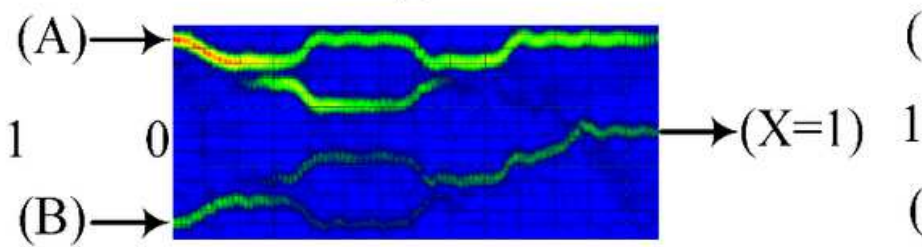

(c)

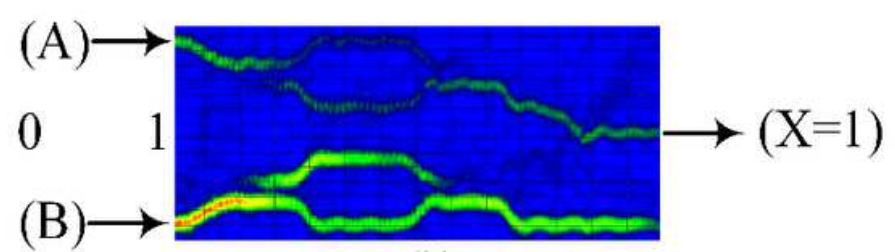

(b)

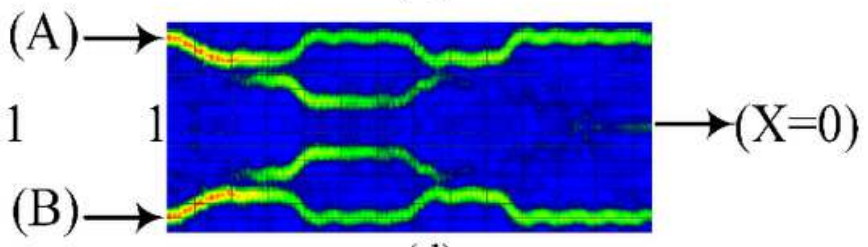

(d)

\section{Figure 4}

Output results for all possible combinations of an optical signal obtained from FDTD
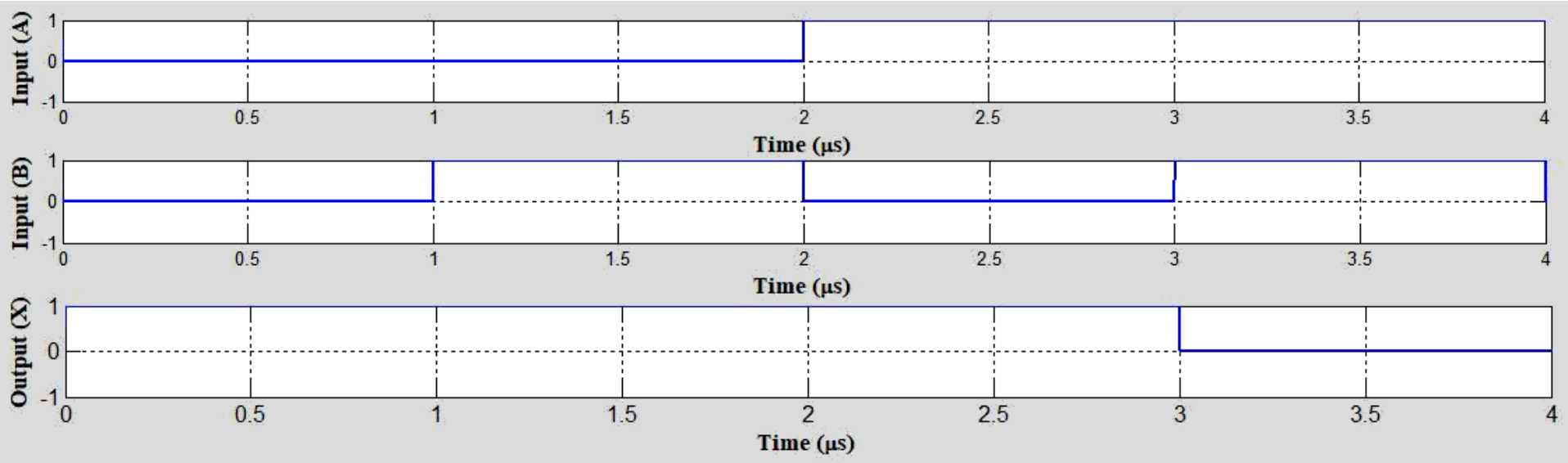

Figure 5

MATLAB simulation results for all-optical NAND gate 


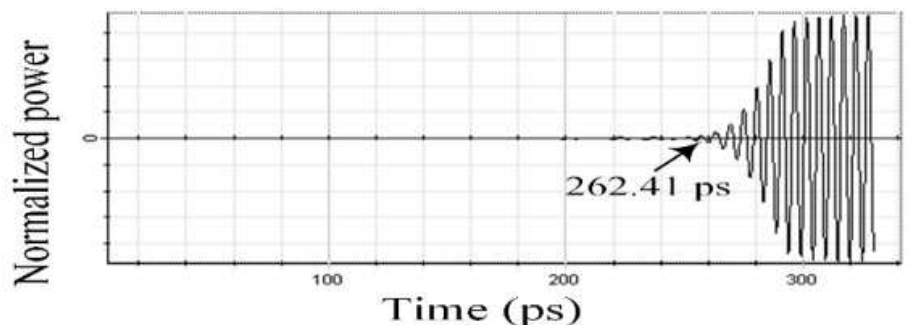

a) "OO"

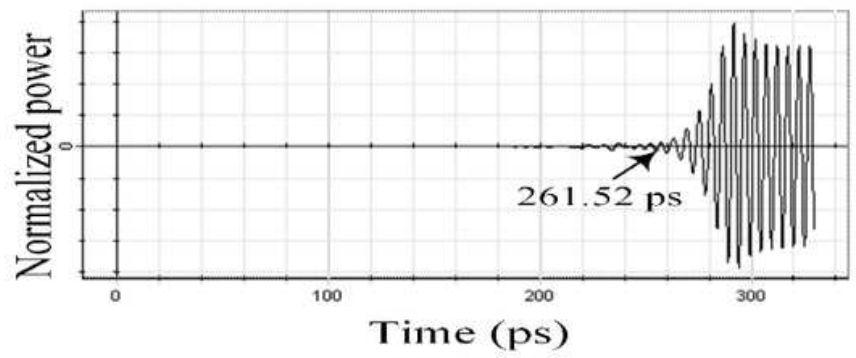

b) "O1"

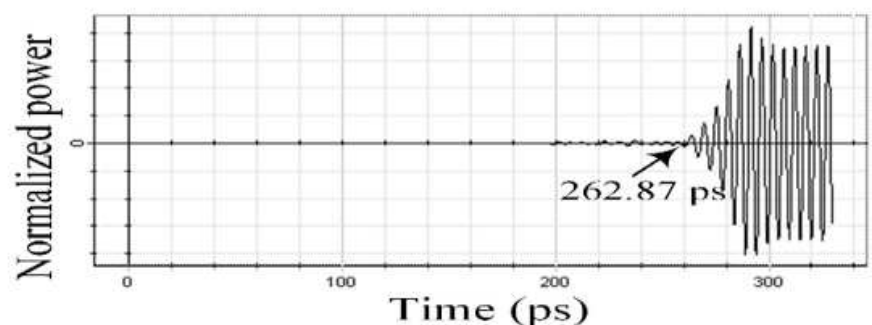

c) $" 10 "$

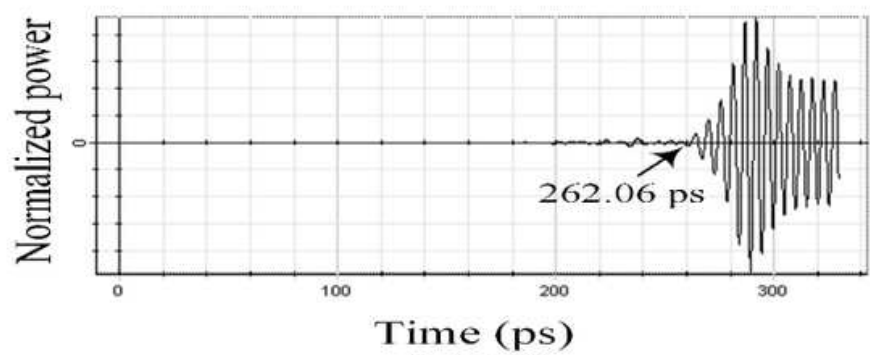

d) " $11 "$

Figure 6

Normalized power versus time for all four combinations: a) "00"; b) “01"; c) "10"; d) "11" 